\title{
Le kiswahili : une politique linguistique d'exception au Tanganyika
}

Aude Chanson

\section{Q OpenEdition \\ 1 Journals}

Édition électronique

URL : https://journals.openedition.org/eastafrica/492

DOI : $10.4000 /$ eastafrica.492

ISSN : 2790-1076

\section{Éditeur}

IFRA - Institut Français de Recherche en Afrique

\section{Édition imprimée}

Date de publication : 1 juillet 2012

Pagination : 49-65

ISSN : 2071-7245

\section{Référence électronique}

Aude Chanson, «Le kiswahili : une politique linguistique d'exception au Tanganyika », Les Cahiers d'Afrique de l'Est / The East African Review [En ligne], 45 | 2012, mis en ligne le 07 mai 2019, consulté le 09 décembre 2021. URL : http://journals.openedition.org/eastafrica/492 ; DOI : https://doi.org/ 10.4000/eastafrica.492 


\title{
Le kiswahili : une politique linguistique d'exception au Tanganyika
}

\begin{abstract}
Aude Chanson
Summary - This article presents the language policy in Tanganyika under German and British colonizations through textbooks. In this territory, the language of instruction was Swahili, an African language used and propagated by Muslim traders, and then European missionaries. Why was English not used as language of instruction in Tanganyika like in any other British colonies? What are the reasons and issues in this area which make it so special? The role of the German colonial administration and missionaries was very important in developing the use of Swahili as vernacular language, and consequently as common language during the British colonial period. The specificity of multi-ethnic society is also fundamental to understand the need of efficient and complete communication with and between populations. This paper explores how Swahili became a lingua franca in Tanganyika under British administration.
\end{abstract}

\section{Introduction}

«Nos ancêtres les Gaulois » n'a pas toujours été la formule appliquée dans toute l'Afrique coloniale. Certes, toutes les colonies en Afrique n'étaient pas françaises, mais ce n'est pas là la seule raison. En effet, lorsque Ernest Lavisse publia en 1884 son manuel d'histoire destiné aux écoliers de France et des colonies, qui fut réédité jusqu'à la fin des années 1950, d'autres puissances coloniales optèrent pour un modèle différent dans leurs colonies. Cet article traite des politiques linguistiques au Tanganyika, un pays qui fut tout d'abord colonisé par l'Allemagne - il appartenait alors à l'Afrique de l'Est allemande (Deutsch-Ostafrika) - puis par le Royaume-Uni - sous l'appellation de Tanganyika Territory. Dans ce large territoire peu peuplé, une langue possédait une tradition écrite avant l'arrivée des Européens, le kiswahili. ${ }^{1}$

On se demandera dans quelle mesure ces politiques linguistiques sont différentes de celles pratiquées ailleurs sur le continent africain à la même époque, et pourquoi. Leur spécificité a-t-elle trait aux acteurs coloniaux qui ont mis ces politiques en place ou sontelles le résultat d'un contexte historique particulier? Le changement de pouvoir colonial a-t-il affecté la place et le rôle accordé au kiswahili, et si oui, de quelle manière ? La

\footnotetext{
${ }^{1}$ Le plus ancien manuscrit découvert en kiswahili date de 1700 et est écrit en caractères arabes (ou Ajami-script, l'alphabet arabe utilisé pour la transcription des langues africaines). Le terme «swahili » est le pluriel du mot arabe sãbil, qui signifie la côte ou la frontière. On peut également le rapprocher de l'adjectif as-sawãhil qui désigne les habitants de la côte. Le préfixe ki- est l'appartenance de classe, ici la langue. Kiswahili signifie alors «la langue de la côte ». A l'arrivée des premiers Européens, la population côtière parle kiswahili. Ce n'est pas le cas des régions de l'intérieur où les populations parlent différents dialectes, bien qu'elles aient été en contact avec la langue swahilie par le biais du commerce caravanier.
} 
politique linguistique, notamment au niveau scolaire, est ici perçue comme une clé de compréhension de dynamiques de développement que l'article s'attachera à explorer.

Pour cela, on s'appuiera sur un manuel publié en 1931, intitulé Masimulizi na mafundisho, dans la colonie britannique du Tanganyika. Tout d'abord, une analyse fine de ce manuel permettra de saisir les enjeux éducatifs et linguistiques sur ce territoire. Dans un second temps, l'action des congrégations religieuses qui sont les premiers acteurs éducatifs au Tanganyika à l'époque coloniale sera examinée. Le rôle des puissances coloniales en matière de politiques scolaire et linguistique sera ensuite traité, ces politiques ayant évolué selon le colonisateur et la période concernée. Enfin, la période qui précède l'indépendance est le moment où la colonie est soumise à de nombreuses réformes ayant pour objectif de préparer la colonie à l'indépendance, en particulier des réformes linguistiques qui reposent sur l'idée que la langue est un élément clé pour la formation d'une identité nationale.

\section{Masimulizi na mafundisho: un manuel de mission de l'entre-deux-geurres}

Le manuel scolaire Masimulizi na mafundisho. Kitabu cha kwanza ("Oral et enseignement. Livre pour débutants»), consulté aux archives missionnaires de Leipzig en Allemagne, était destiné aux élèves des missions luthériennes du nord du Tanganyika. En effet, les missions chrétiennes étaient les acteurs les plus importants en matière de politique scolaire au Tanganyika pendant toute la période coloniale, aussi bien allemande que britannique. Surtout après la Première Guerre mondiale, les missions installèrent dans la colonie des imprimeries afin de publier plus facilement aussi bien des manuels scolaires pour leurs écoles que des livres de chants religieux ou tout autre matériel nécessaire au bon fonctionnement de leurs établissements et à la diffusion de leur enseignement religieux et scolaire. Ceci peut s'expliquer par le fait que la technologie et les produits étaient plus accessibles financièrement, mais aussi parce que les documents imprimés n'étaient conçus que pour un espace limité et une audience particulière. En effet, si la publication avait été éditée en anglais et destinée à une grande audience, il aurait été plus facile de l'imprimer en grande quantité en Grande-Bretagne puis la faire livrer en Afrique de l'Est. Mais le manuel étudié ici est rédigé en kiswahili, langue véhiculaire $^{2}$ du Tanganyika et langue vernaculaire sur la côte est-africaine. Il ne concerne donc pas toutes les colonies de l'Empire britannique, mais une seule colonie, et plus précisément une région - le Lushoto - et une mission, Lwandai, située à environ 90 kilomètres de Tanga au nord-est du Tanganyika, à la frontière avec le Kenya. Par conséquent, le public et de fait le nombre d'exemplaires sont limités.

Il est important de souligner que les manuels scolaires interprètent les programmes scolaires (à la différence du curriculum). Les auteurs des manuels transcrivent de manière implicite ou explicite leur vision des colonisés, des colonisateurs et des rapports que ceux-ci entretiennent avec les colonisés. Les manuels reflètent donc l'idéologie de leurs auteurs et permettent aux chercheurs de saisir les valeurs de leur époque.

\footnotetext{
${ }^{2}$ Une langue véhiculaire est une langue de communication; elle se distingue de la langue vernaculaire qui est la langue maternelle des interlocuteurs.
} 
Cet ouvrage de 150 pages est rédigé entièrement en kiswahili et non en anglais, en vue d'être compris et d'intéresser les apprenants. Les missions chrétiennes d'Afrique, et plus particulièrement en Afrique de l'Est, développèrent le plus possible l'enseignement et l'alphabétisation des populations locales dans la langue maternelle des élèves afin de ne pas les acculturer. ${ }^{3}$ En effet, l'école était souvent perçue comme un lieu de dépossession de la culture africaine éloignant les élèves de l'ordre traditionnel - un processus qu'il convenait d'éviter selon les missionnaires. La scolarisation dans la langue maternelle avait donc pour objectif de limiter l'acculturation en créant un lien fort entre les colonisés et les missionnaires. Dès la fin du XIX ème siècle, des travaux furent menés par des inspecteurs des missions dans les colonies allemandes mais également dans les colonies anglaises et françaises qui démontrèrent que l'enseignement effectué en langue maternelle donnait de meilleurs résultats. Lors d'une conférence internationale des missions, l'inspecteur des missions Zahn déclara que :

"Wenn man noch hoffen kann, ein geschlossenes Volk qu christianisieren und Gemeinden zu gründen, die imstande sind, das Christentum weiterzubetragen, so ist es seine Vorbedingung für diese Aufgabe, den Eingeborenen eine nationale Erziehung, d.h. in ihrer Muttersprache zu geben.»4

Ce manuel est imprimé en noir et blanc avec une reliure cartonnée verte. On compte vingt-huit gravures et huit photographies qui s'attachent toutes à illustrer l'environnement dans lequel évoluent les élèves. Mais cette iconographie n'est qu'un complément d'information pour le lecteur: elle permet de donner une représentation des choses, et non de les définir. On en déduit que cet ouvrage est destiné à un public déjà avancé dans son enseignement. Les phrases sont longues et de structures complexes, ce qui est en contradiction avec le sous-titre «Kitabu cha kwanza » qui signifie «premier livre », c'est-à-dire livre pour débutants. On peut alors supposer que ce livre est à l'attention des élèves séminaristes qui possèdent déjà un bon niveau en kiswahili mais qui commencent leur apprentissage religieux.

Au niveau du contenu, le livre est construit en cent-quatorze chapitres. Il n'est pas nécessaire ici de les énumérer tous. On peut relever certains thèmes centraux qui mettent en lumière ce que les missionnaires veulent enseigner. Dès le sommaire, un constat s'impose: l'importance de la faune et de la flore. En effet, plus de trente chapitres sont consacrés aux animaux que l'on rencontre au Tanganyika : des éléphants aux hippopotames en passant par les buffles, les hyènes mais aussi les serpents, les chiens, les insectes ou autres animaux de la ferme. En ce qui concerne la flore, cinq chapitres sont dédiés aux arbres comme le baobab et le bananier ainsi qu'aux plantes et aux fruits tels que le maïs et la noix de coco. La nature est également présente avec des chapitres sur les lacs et les rivières, ainsi que les montagnes. Le manuel se situe vraiment dans une géographie et un environnement spécifiques. Ce n'est pas n’importe quelle montagne qui est évoquée ici, mais le Kilimandjaro, le plus haut sommet d'Afrique, qui

\footnotetext{
${ }^{3}$ L'acculturation résulte d'un contact continu et direct entre des groupes d'individus de cultures différentes et entraine des modifications dans les modèles culturels initiaux d'un des groupes. Le terme d' "acculturation " est apparu en 1880 chez Powell pour décrire la perte de la culture d'origine mais aussi l'appropriation d'une nouvelle culture (Cuche, 2001).

${ }^{4}$ Bundesarchiv, DKG, Berlin, Bd. Nr. 977, p. $76:$ «Si on peut encore christianiser un peuple renfermé et fonder des communautés capables d'apporter le christianisme, alors il y a une condition préalable pour cette mission qui est de donner une éducation nationale aux indigènes, c'est-à-dire dans leur langue maternelle ».
} 
se situe à proximité de la station. Tous ces exemples nous montrent bien comment les missions parlent à leurs élèves d'une réalité qui les entoure et non d'un idéal lointain. Ils ancrent leurs cours et l'apprentissage du vocabulaire dans un environnement familier et évocateur pour les élèves mais aussi pour les assistants et les maittres. En effet, étant donné le manque patent de missionnaires enseignants, les missions ont dû former des enseignants africains. Il est alors important de donner à ces derniers des outils qu'ils puissent s'approprier. Les connaissances approfondies des missionnaires du milieu traditionnel africain et leurs compétences linguistiques les rendent capables de rédiger des outils pédagogiques adaptés, à la différence d'autres acteurs éducatifs, particulièrement le gouvernement colonial.

Les chapitres sur les différents groupes ethniques sont également révélateurs de l'intérêt porté par les missionnaires aux sociétés africaines. En effet, dès leur arrivée, les missionnaires ont mené un important travail de documentation linguistique, géographique, scientifique et ethnographique. Avant même d'établir certaines missions, des missionnaires partaient en expédition pour mener des études de terrain. Bien sûr, il s'agissait de savoir quelles pouvaient être leurs chances de réussite dans l'implantation d'une mission, mais la méthode de travail utilisée par les missionnaires et les écrits qu'ils en ont tirés restent jusqu'à aujourd'hui une précieuse source d'informations, riche et détaillée, pour les historiens. Tous ces points d'intérêt se retrouvent dans ce manuel qui, d'une part, n'est pas rédigé dans une langue européenne mais africaine, le kiswahili, et d'autre part met en avant la culture et la richesse d'un territoire. La diversité de sa faune et de sa flore est un atout que les missionnaires soulignent tout au long de l'ouvrage. Autrement dit, ils ne cherchent pas ici à inférioriser cette région, ni à prouver la supériorité des puissances coloniales sur les populations africaines, mais au contraire à montrer qu'il n'y a pas une population africaine unique et homogène, comme beaucoup d'Européens le laissaient croire, mais des populations africaines plurielles et différentes ayant chacune leur histoire et leurs traditions. Une douzaine de chapitres décrit différents groupes ethniques tels que les Wahehe, les Wanyasa, les Wachagga, les Wasambaa et les Masais. Ces peuples vivent dans différentes régions de la colonie et des gravures illustrent leur costume, leur coiffure etc. afin de permettre aux élèves de les typifier et les identifier.

À la lecture de ce manuel scolaire, on peut se poser la question de la représentation de la présence de la civilisation européenne et de la colonisation sur le territoire du Tanganyika. Aussi curieux que cela puisse paraittre, la colonisation britannique ou allemande y est en effet quasiment absente. Seuls deux chapitres y font clairement référence : «Wazungu wa Usambaa na kazi zao » (le travail des blancs de l'Usambaa) et "Johannes Rebmann», un explorateur et missionnaire allemand ${ }^{5}$. Ces deux chapitres portent sur les Européens mais ils montrent uniquement les relations et les interactions de ces derniers avec les Africains, sans refléter les hiérarchies et les rapports de force à l'œuvre.

${ }^{5}$ Johannes Rebmann (1820-1876) est un explorateur et missionnaire allemand de la mission protestante de Bâle qui est considéré comme le premier Européen, avec Johan Ludwig Krapf, à découvrir le Kilimandjaro et le mont Kenya. Il apprit plusieurs langues africaines et rédigea un dictionnaire en kiswahili (Paas, 2011). 
À d'autres endroits, on trouve des explications sur des technologies importées par les Européens comme le train (gari la moshi), les aménagements portuaires (bandari za Tanga na Dar-es-Salaam), ou encore le bateau à vapeur (meli). Mais toutes ces explications font partie intégrante du processus de découverte et d'apprentissage de la société qui entoure les jeunes élèves. Lorsque paraît ce manuel en 1931, le Tanganyika, alors colonie britannique, est doté d'une infrastructure de transport relativement bien développée. Les informations diffusées dans ce manuel sont donc utiles aux jeunes élèves qui seront amenés à voir et peut être même à utiliser ces équipements.

Quant au travail et au rôle de christianisation des missions, ils ne sont étonnement que peu abordés. En effet, cet ouvrage, alors même qu'il s'agit d'un travail rédigé, imprimé et utilisé par des missionnaires, ne fait ni l'apologie de la religion chrétienne ni de la conversion des populations. On n'y trouve aucun message prosélyte. Dans tout l'ouvrage, on ne fait mention qu'une fois d'une église chrétienne et que très rarement des missions religieuses présentes sur le territoire. Pourtant, alors même qu'elles sont quasiabsentes de ce manuel, les missions chrétiennes jouèrent un rôle de premier plan dans la politique scolaire et linguistique de la colonie du Tanganyika.

\section{Les congrégations religieuses : premiers acteurs éducatifs pendant la colonisation européenne au Tanganyika}

On peut dater la première évangélisation en Afrique de l'Est à la fin de l'année 1860 lorsque trois prêtres français appartenant à la congrégation catholique des Pères du Saint-Esprit atteignirent la côte swahilie en passant par l'archipel de Zanzibar, accompagnés de six infirmières, un médecin et plusieurs artisans - l'ensemble sous l'égide de l'évêque Maupoint de l'île de la Réunion. Le succès de cette première expédition fut concrétisé par l'envoi d'autres missionnaires quelques années plus tard, notamment à Bagamoyo, un village situé sur la côte du Tanganyika (Markmiller, 1995).

Les Pères Blancs ${ }^{6}$, autres membres d'une congrégation française, arrivèrent dans la région des Grands Lacs en 1878. Les suivirent les moines bénédictins des monastères allemands de Saint-Ottilien en 1887. Les premiers missionnaires furent donc des catholiques français mais ils furent rapidement, et en nombre, rejoints par les missionnaires allemands puis britanniques et enfin américains. En 1891 s'installèrent les premières missions protestantes allemandes comme les missions de Berlin (Berliner Mission), de Béthel (Bethel-Mission), les Frères moraves (Herrnbuter Brüdergemeine) puis un peu plus tard, en 1893, les missions de Leipzig (Evangelische-Lutherische Mission zu Leipzig). En effet, avec l'annonce en 1885 par le chancelier allemand de l'Empire, Otto von Bismarck, de la colonisation de l'Est africain, les missionnaires protestants, et de surcroît allemands, se sentirent au Tanganyika chez eux et en droit d'y propager l'évangile, recevant l'autorisation d'entamer leurs activités religieuses avant les missionnaires catholiques allemands (1892) (Schmidlin, 1913).

\footnotetext{
${ }^{6}$ L'origine de l'appellation de Pères «Blancs » provient du fait que ces missionnaires étaient vêtus de blanc, portant le plus souvent une longue robe à la romaine. Ils se considéraient comme une société d'inspiration romaine catholique.
} 
On peut noter l'absence d'unité parmi les missionnaires qui se partagent l'Afrique de l'Est en fonction de leur provenance et de leur nombre. On constate alors, de manière générale, que les missionnaires catholiques s'installent aux bords des lacs Victoria et Tanganyika tandis que les missionnaires protestants s'établissent dans les districts de Tanga, Pangani et Wilhelmstal, ${ }^{7}$ ainsi qu'aux environs du lac Nyassa.

Dès leur implantation, les missions jouèrent un rôle important auprès des populations africaines. Comme indiqué précédemment, les missionnaires menèrent de nombreuses études sur les populations colonisées. Ils cherchèrent le dialogue et établirent dans la mesure du possible une relation de confiance avec les populations environnantes. Le gouvernement allemand saisit rapidement l'intérêt du travail des missions et leur confia la tâche d'apporter " la culture et la civilisation aux Africains » (Acker, 1909), notamment grâce au travail scolaire mené dans les stations. La « civilisation » dans son ensemble devait être amenée aux colonisés pour qu'ils atteignent "l'élévation pour le corps et l'âme»(Acker, 1909). Il n’était pas considéré comme possible pour les Africains d'être accomplis sans d'abord intégrer l'éthique d'obéissance et de performance individuelle des Européens (Tetzlaff, 1984). Les missions ont également pris en charge la tâche centrale de maintenir la paix entre les différents groupes de population de la colonie, et de les initier à une nouvelle autorité. L'administration coloniale avait alors conscience que les missions étaient seules à pouvoir mener à bien cette action, dans le temps et l'espace, compte tenu de leur présence ancienne et au plus près des populations. Lorsque Schmidlin écrit que :

"Die Mission ist es, die unsere Kolonien geistig erobert und innerlich assimiliert, soweit eine solche Assimilation in Anbetracht der tiefgreifenden Verschiedenheiten überhaupt durchfübrbar ist. Der Staat vermag die Schutrgebiete sich wohl äußerlich an- und einzugliedern; das tiefere Ziel der Kolonialpolitik, die innere Kolonisation, muss ibm die Mission vollbringen helfen. Durch Strafen und Gesetze kann der Staat den physischen Gehorsam erzwingen, die seelische Unterwürfigkeit und Anhänglichkeit der Eingeborenen bringt die Mission zustande 》 (Schmidlin, 1913), 8

il signifie que les missions sont un outil de colonisation. Et il ne s'agit pas seulement de l'action pacificatrice menée par les missions. Cette citation a trouvé un large écho dans les milieux des congrégations religieuses de l'époque. Le travail des missions en coopération avec l'Etat devait être encouragé pour le «bien-être des colonies » (Schwager, 1912). La conquête mentale et l'assimilation de l'intérieur sont ainsi devenues les lignes directrices du programme des missions en Afrique de l'Est (Gründer, 1982). Aussi l'administration s'est efforcée de soutenir les sociétés missionnaires lors de la première phase de la colonisation - de 1885 à 1905. L'administration a considérablement facilité la création de stations missionnaires, toujours dans le but de pacifier? les populations locales. Les missions n'étaient pas opposées à jouer ce rôle de

\footnotetext{
${ }^{7}$ Wilhelmstal est l'ancien nom allemand de la ville de Lushoto située au nord-est de l'actuelle Tanzanie.

8 «C'est la mission, qui conquiert mentalement et assimile de l'intérieur nos colonies, rendant une telle assimilation possible malgré des différences profondes. L'Etat préfère annexer et intégrer les protectorats de l'extérieur; l'objectif plus profond de la politique coloniale, la colonisation interne, doit aider à réaliser celui des missions. Par l'intermédiaire des punitions et des lois, l'Etat peut forcer à l'obéissance physique, qui crée la soumission et la dépendance des indigènes vis-à-vis des missions ».

${ }^{9}$ La pacification dans le contexte colonial signifie le contrôle des habitants afin d'éviter toutes contestations susceptibles de troubler l'ordre public colonial.
} 
médiateurs, notamment lorsque les fonctionnaires coloniaux leurs offraient des avantages tels que des terrains, des outils et du matériel, mais aussi la possibilité d'emprunt d'argent auprès des campements militaires. Du point de vue des missionnaires, la participation à la politique d'apaisement pouvait aussi se justifier par des raisons humanitaires. C'était pour elles le seul moyen de s'occuper des vaincus et de s'assurer de leur sort. L'administration a accordé une grande importance à la coopération avec les missions, notamment au vu de l'ensemble des privilèges accordés par l'administration aux missions. De cette façon, une partie des dépenses des congrégations pour la formation et l'assistance médicale était déjà prise en charge. Un aspect encore plus important aux yeux des missionnaires était la reconnaissance de leur travail par le gouvernement colonial.

De plus, les missionnaires jouaient un rôle certain auprès des autorités coloniales dans la mesure où ils n'effectuaient pas autant de mutations que les fonctionnaires coloniaux expatriés. Par conséquent, les missionnaires possédaient une meilleure connaissance du terrain et de la population que le colonisateur. Pendant la révolte MajiMaji en $1905^{10}$, il était par exemple possible pour les missionnaires de distinguer les personnes dignes de confiance des insurgés. Ceci fut très utile lors de l'arrivée des troupes coloniales envoyées pour réprimer la révolte (Niesel, 1971).

Une particularité de la colonie d'Afrique de l'Est allemande était le fait que la plupart des sociétés missionnaires se sont adaptées aux directives du gouvernement colonial, notamment en ce qui concerne l'introduction du kiswahili. En effet, l'administration a très vite décidé l'instauration du kiswahili comme langue véhiculaire alors que les missionnaires l'avaient d'abord rejetée dans la mesure où le kiswahili était considéré comme la langue des musulmans de la côte. En effet, on compte environ 40\% des mots d'origine arabe en kiswahili; ce chiffre augmente chez les populations côtières ou à Zanzibar mais diminue à l'intérieur du territoire. Les congrégations religieuses voyaient se dessiner de la part de l'administration coloniale une politique pro-arabe dans le domaine de l'éducation - une crainte alimentée par l'introduction de cours sur l'islam enseignés dans les écoles gouvernementales (van der Ploeg, 1977).

Cependant, cette position de rejet du kiswahili évolua pour éviter que les élèves quittent les stations pour les écoles gouvernementales. La maîtrise du kiswahili était en effet une condition indispensable pour le travail de collaborateur dans l'administration. Les missions ont donc dû s'organiser de manière à satisfaire les exigences du gouvernement et par là même obtenir des subventions (Tetzlaff, 1984). Les missionnaires allemands et anglais mais également français menèrent un énorme travail linguistique, non seulement un travail d'enseignement mais aussi un travail destiné à faire évoluer la langue. Ils créèrent de nouveaux concepts pour s'exprimer, particulièrement en matière religieuse mais pas uniquement. Ils swahilisaient des mots allemands ou

\footnotetext{
${ }^{10}$ La révolte Maji-Maji fut le seul véritable soulèvement à l'encontre de la puissance coloniale allemande entre 1905 et 1906. Elle fut déclenchée lors de la rude sécheresse de l'hiver 1904-1905 par plusieurs tribus d’Afrique de l'Est allemande. Les rebelles étaient peu armés face aux fusils allemands mais se croyaient protégés par les potions des sorciers traditionnels, qui devaient transformer les balles en eau (maji en kiswahili). Les combats étaient meurtriers mais les insurgés nombreux. Le gouverneur von Götzen dut faire appel à l'Empereur pour envoyer du renfort au plus fort de la révolte. Cet événement violent étouffa toute autre idée de rébellion en Afrique orientale allemande jusqu’à la Première Guerre Mondiale (Niesel, 1971).
} 
encore latins afin d'être plus aisément compris par les populations africaines. Il est important de souligner que Ludwig Krapf (compagnon de Johannes Rebmann), missionnaire allemand de la Church Missionary Society rédigea en écriture latine la première grammaire swahilie en 1850 et le premier dictionnaire swahili en 1882. En effet, l'un des premiers travaux entrepris par les missionnaires fut la latinisation de l'écriture swahilie, à l'origine écrite en caractères arabes. Ces travaux furent suivis de centaines d'autres pendant toute la colonisation allemande. Le kiswahili devint alors la langue de communication au sein de la colonie aussi bien entre les populations locales qu'avec l'administration et entre les missionnaires. Les missionnaires en particulier favorisèrent de manière considérable l'utilisation du kiswahili et des différentes langues locales (Mirbt, 1914). Les Africains étaient souvent étonnés de voir des missionnaires discuter entre eux en kiswahili alors même qu'ils étaient Européens. En effet, les congrégations religieuses d'une même mission étaient le plus souvent internationales; on comptait en moyenne plus de six nationalités différentes. Afin de faciliter la communication, le kiswahili devint donc la langue commune. ${ }^{11}$

Remplissant les exigences de la puissance coloniale pour la diffusion du kiswahili, l'administration favorisa l'emploi de fonctionnaires chrétiens, notamment par l'intermédiaire d'une résolution votée au Parlement à Berlin en 1900, et limita l'éducation formelle des musulmans aux écoles gouvernementales, tout en introduisant des missionnaires dans ces nouvelles écoles - il ne faut pas oublier le fait qu'à côté de la Landessprache, ${ }^{12}$ seul l'allemand pouvait être enseigné. ${ }^{13}$ Mais les députés partirent du principe que les musulmans constituaient une menace pour le travail des congrégations religieuses et les écoles gouvernementales se trouvaient dans les régions côtières où il n'y avait pas ou peu d'écoles missionnaires chrétiennes. Par conséquent, lorsqu'il fallut employer des missionnaires dans les nouvelles écoles gouvernementales, les difficultés commencèrent compte tenu du manque de missionnaires sur place. Cela posa le problème de la concurrence entre les deux principales confessions chrétiennes: catholique et protestante. En outre, la notion de Landessprache est trompeuse dans la mesure où il n'y a pas en Afrique de l'Est de langue nationale au sens européen du terme. L'administration coloniale a instauré le kiswahili comme langue véhiculaire tandis que les missionnaires enseignèrent les Stammessprachen ${ }^{14}$, c'est-à-dire les langues ethniques. Malgré toutes les erreurs et les fausses suppositions, le gouvernement à Berlin souhaitait que les missions forment des auxiliaires swahilophones. Certaines missions, comme les Bénédictins, réagirent favorablement face à ces attentes :

"... dass die Forderung einer besonderen Vorbildung für farbige Beamte eine gerechtfertigte ist, und dass bisher die Missionsschulen diese spezielle Vorbildung nicht gewährten. Um jedoch die Ausfïbrung des vorgenannten Reichstagsbeschlusses ibrerseits zu ermöglichen, um ferner ibren Missionsscbülern die Möglichleeit zu verschaffen, Fortkommen und Stellung zu finden, endlich um dem von der Mission schwer empfundenen Zustande abzubelfen, dass die eingeborenen Cbristendörfer durch nicht-christliche eingeborene Unterbeamte vervaltet und kontrolliert

\footnotetext{
11 «MPAPUA an Gouvernement », 26.10.1901, in TNA, G 9 /24, p. 151.

${ }^{12}$ Landessprache signifie en français la langue du pays, c'est-à-dire la langue nationale.

13 «Stenographische Berichte über die Verhandlungen des Reichstags. 10. Legislaturperiode. 1. Session. 1898/1900. Fünfter Band. Berlin, 1900, 4080 f. (Inhalt der Resolution), 4087 (Verabschiedung) », in Eggert (1970).

14 Stammessprachen signifie littéralement en français les langues des tribus, à savoir les dialectes.
} 
werden, will die Mission durch Erweiterung und Anpassung des Lebrplanes auch in ibren Schulen Zöglinge heranbilden, die den als Beamte an sie zu stellenden Anforderungen entsprechen. Sie will daher ibren Lehrplan gemäß dem der Gouvernementschulen ergänzen und bittet um Mitteilung desselben. „15

La déclaration centrale de cet accord fut l'adaptation des écoles des missions au programme d'études des écoles gouvernementales. Les Bénédictins, par exemple, qui ont suivi les souhaits du gouvernement dans le domaine de la formation des chrétiens africains, ont obtenu en contrepartie qu'aucune nouvelle école gouvernementale dans le secteur des missions bénédictines ne soit installée et que le gouvernement modère ses prises de décisions en faveur de l'islam. Pour bénéficier également des avantages tels que certaines subventions ou priorités, les missions protestantes suivirent également au fur et à mesure le programme d'études des écoles gouvernementales (Gründer, 1982). Mais l'emploi d'élèves chrétiens dans l'administration coloniale restait encore quasi-inexistant sous la colonisation allemande. Cela peut s'expliquer par le faible taux de scolarisation des congrégations jusqu'en 1910 (Schäppi, 1937).

Le début de la colonisation montre donc une volonté d'unification à travers une langue commune de ce large territoire qui formait une mosaïque ${ }^{16}$ de langues et de cultures difficile à contrôler. On assista donc à une swahilisation du territoire. Cette politique fut étendue après la Première Guerre mondiale, alors que la Grande-Bretagne fut désignée par la toute jeune Société des Nations (S.D.N.) pour prendre en charge cette colonie (Porte, 2006). Mais cette dernière ne se sentait pas très concernée par cette acquisition. Par conséquent, le changement de pouvoir colonial ne changea pas réellement la politique linguistique des missions chrétiennes, lesquelles continuèrent à promouvoir le kiswahili comme lingua franca ${ }^{17}$ de cette zone.

Par ailleurs, le gouvernement colonial britannique expulsa tous les ressortissants allemands du Tanganyika après la Première Guerre mondiale, y compris les missionnaires allemands. Des centaines d'écoles se retrouvaient alors sans professeurs. Les congrégations anglaises présentes en Afrique de l'Est à l'époque de la colonisation étaient: l'Église de la Société missionnaire de Londres (Church Missionary Society in London); la Mission des universités pour l'Afrique centrale (Universities' Mission to Central Africa - UMCA); la Société missionnaire de Londres (London Missionary Society). L'administration coloniale britannique confia à ces congrégations un rôle fondamental dès le début des années 1920, reprenant certaines missions allemandes désertées. Elles

\footnotetext{
15 « (...) que l'exigence d'une formation particulière pour les fonctionnaires de couleur est justifiée et que jusqu'ici les écoles des missions n'accordaient pas cette formation particulière. Cependant, pour pouvoir appliquer le décret parlementaire, pour procurer aux élèves des missions la possibilité de progresser, de trouver une position, enfin pour que les missions remédient aux difficiles conditions, que le village chrétien indigène soit administré et contrôlé par des fonctionnaires indigènes non-chrétiens, la mission veut former grâce à une extension et une adaptation de son programme d'enseignement dans ses écoles des élèves qui correspondent aux exigences pour être fonctionnaires. Elle veut donc compléter son programme d'études conformément à celui des écoles gouvernementales et demande de le communiquer", in "Protokoll der Verhandlungen zwischen stellvertr. Gouverneur Dr. Stuhlmann und P. M. Hartmann, Präfekt der Missionsgenossenschaft der Benediktiner, 16.11.1900», in Eggert (1970).

${ }^{16}$ L'Afrique de l'Est allemande est une colonie qui regroupe plus de 120 groupes ethniques.

${ }^{17}$ Le terme de lingua franca vient de l'italien et signifie une langue commune à deux interlocuteurs ayant des langues maternelles différentes. Les lingua francas se sont souvent développées pour des raisons commerciales, mais aussi diplomatiques ou administratives.
} 
jouèrent un rôle de premier ordre dans la mise en place d'un système colonial éducatif dans la mesure où elles étaient les principales interlocutrices du nouveau gouvernement colonial. Cependant, elles ne pouvaient faire face seules au manque de professeurs. Par conséquent, il fallut faire appel aux congrégations françaises. Plusieurs témoignages font part d'un manque manifeste d'enseignants capables d'enseigner l'anglais dans les écoles missionnaires à l'époque coloniale britannique. ${ }^{18}$ Dans cette région où se côtoyaient de nombreuses langues et dialectes, la question du kiswahili et de son rôle dans la société africaine du Tanganyika apparaît comme primordiale. Très rapidement, les congrégations aussi bien britanniques que françaises ont défendu l'usage du kiswahili comme langue d'enseignement en Afrique de l'Est, exception notable puisque les Anglais avaient pour habitude d'enseigner en anglais dans leurs colonies. Le Tanganyika constitue donc une exception dans ce domaine où les missionnaires ont joué un grand rôle. Il se pose alors la question du rôle joué par les puissances coloniales au niveau de la politique linguistique de ce territoire.

\section{Quel rôle pour les puissances coloniales dans les politiques linguistiques ?}

En Afrique, les puissances coloniales firent face à une multiplicité de dialectes qui rendait difficile la communication avec les populations locales. Le modèle français prônait, dans ce contexte, un universalisme monolingue colonial, le français - qui resta parfois contrarié par des contraintes locales - afin d'unir l'ensemble de la population africaine comme en Afrique Occidentale Française (AOF) et en Afrique Equatoriale Française (AEF), tandis que le gouvernement colonial allemand choisit en Afrique de l'Est de diffuser le plus largement possible également une même langue, mais une langue africaine - le kiswahili. Il est intéressant de noter que le colonialisme allemand se distingue de ce fait du colonialisme français, défini comme «glottophage» par le linguiste Louis-Jean Calvet (Calvet, 1974) dans la mesure où la langue française est, à quelques exceptions près, la seule langue autorisée dans la plupart des écoles coloniales sous domination française. Le discours colonial cherche à montrer que «la glottophagie est un fait d'évidence (...) et de plus souhaité par les colonisés eux-mêmes » (Calvet, 1974). Cela s'explique par la volonté d'unifier les colonies en effaçant tout particularisme, dont les langues font partie. Il s'agit d'un impérialisme de la langue française propre au système colonial français, qui associe langue et degré de civilisation : le « civilisé » parle le français tandis que le «sauvage » s'exprime dans un dialecte, perçu comme une «sous-langue ». À l'époque coloniale, la linguistique hiérarchisait également les langues, servant par là-même l'ordre colonial - supériorité de l'Occident sur les peuples africains - dans la mesure où les langues indo-européennes étaient considérées comme les plus évoluées. Selon les colonisateurs, cet apprentissage obligatoire du français reposait sur deux grands principes qui sont: "l'intérêt du colonisé afin d'accéder à la civilisation»; l'incapacité des dialectes à remplir cette mission de civilisation comme le montre par exemple selon les colonisateurs l'incapacité de véhiculer des concepts scientifiques (Calvet, 1974). C'est pour cette raison que les

\footnotetext{
${ }_{18}$ Archives des Pères du Saint-Esprit, 2K1.
} 
manuels scolaires dans l'empire colonial français étaient exclusivement rédigés en français et centraient toutes leurs leçons sur la France et sa puissance - économique, sociale et technologique - comme le montre la collection africaine des Mamadou et Bineta en AOF au début des années 1930 (Davesne, 1931).

La diffusion d'une langue africaine sur l'ensemble du territoire est certes une marque d'adaptation, mais elle peut également être perçue comme le résultat d'un manque d'intérêt, d'un désinvestissement voire d'une crainte de la part du colonisateur de voir le colonisé apprendre la langue du blanc. En effet, certains craignaient que la maîtrise de la langue du colon puisse devenir une "arme ${ }^{19}$ dans les mains des colonisés. Un compromis a donc été trouvé, comme au Tanganyika, où les élites suivaient leurs enseignements dans la langue coloniale - allemande puis anglaise - et le reste de la population en langue africaine.

Comme mentionné précédemment, les relations entre les gouvernements coloniaux et les missions religieuses varient selon les époques, mais il existait un cadre juridique qui imposait une politique linguistique dans toutes les écoles. Dans la loi du Protectorat du 10 septembre 1900 et notamment le paragraphe 14, les missions obtinrent la liberté et le droit de culte ainsi que la possibilité de créer des édifices religieux. Cette nouvelle législation succéda à la conférence de Berlin, qui s'était déroulée du 15 novembre 1884 au 26 février 1885 et qui prévoyait la liberté de conscience et la pratique des cultes dans l'ensemble des colonies des pays signataires (Spellmeyer, 1931). Cette conférence, initiée par le Portugal et organisée par l'Allemagne, avait réuni les principaux pays concernés par la colonisation. La législation coloniale ne changea pas fondamentalement les conditions de vie des missions et de leurs écoles. Seule la mention à propos de l'apprentissage d'autres langues étrangères, comme le français ou l'anglais par exemple, modifia réellement les programmes/syllabus des écoles missionnaires.

En effet, si une langue étrangère était enseignée dans ces écoles, cela devait être obligatoirement l'allemand pour le cas de l'Afrique de l'Est allemande. Ce nouveau règlement s'explique par le désir toujours plus fort de la part du gouvernement à Berlin, avant la Première Guerre mondiale, de diffuser la langue allemande dans les colonies, alors que sur place l'administration coloniale reste persuadée que l'introduction du kiswahili comme langue véhiculaire est plus adaptée en Afrique orientale. Le kiswahili est donc resté la langue d'enseignement dans les écoles missionnaires tandis que l'allemand le devenait dans les classes supérieures des écoles gouvernementales. L'administration proposa aux écoles des missions d'importantes subventions pour favoriser et inciter l'introduction de l'allemand comme langue d'enseignement : primes, fournitures et matériel scolaires (von König, 1913). Mais les capacités données aux missionnaires étaient limitées dans la mesure où ils enseignaient déjà une langue vernaculaire et le kiswahili. La plupart des écoles se trouvaient en brousse avec un matériel limité et une présence très irrégulière des élèves. Il était déjà difficile d'envisager des cours uniquement en kiswahili, alors l'apprentissage de l'allemand, malgré les subventions, était considéré comme irréaliste par les missionnaires. Seuls les Bénédictins

\footnotetext{
${ }^{19}$ La maîtrise de la langue française permettait d'avoir accès à toute la littérature française et notamment aux concepts de la révolution française. Une contestation intellectuelle de l'ordre colonial pouvait donc naittre à partir de l'enseignement des colonisés (Calvet, 1974).
} 
tentèrent l'expérience compte tenu de leur avance sur l'adaptation de leur programme d'études avec celui du gouvernement (Eggert, 1970).

Cette différence de point de vue entre le gouvernement central de Berlin et les missionnaires sur place, allemands mais également français ou anglais, engendra une cristallisation des relations entre les deux parties. En effet, les objectifs dans le domaine de l'instruction n'étaient pas nécessairement communs. D'une part, les écoles gouvernementales avaient pour but de former de bons et loyaux sujets de l'Empire allemand, tandis que les écoles missionnaires cherchaient à évangéliser. La connaissance de la langue allemande était indispensable dans les écoles coloniales pour former, entre autres, les futurs fonctionnaires de la colonie. Les missionnaires, à l'inverse, favorisaient fortement l'apprentissage de la langue maternelle et du kiswahili, tandis que l'administration ne perdait pas de vue qu'elle:

«(...) muss mit allen Mitteln dabingestrebt werden, dass die Eingeborenen Deutsch als Haupt- und Umgangssprache lernen, denn diese ist das deutlichste Zeichen der Herrschaft.» (Meinhof, 1906) ${ }^{20}$

Alors même que le gouvernement colonial et les missions chrétiennes devaient travailler main dans la main, l'arrivée de l'Empereur Guillaume II ne permit plus aux missions d'être les équivalents de l'administration coloniale dans de nombreux domaines par rapport à l'Etat. Ce dernier investit beaucoup plus d'argent et d'hommes pour accomplir les devoirs régaliens dans la colonie souhaitant faire de l'Afrique de l'Est allemande un territoire allemand. Mais la Première Guerre mondiale détruisit ses plans.

Evidemment, avec le changement de pouvoir colonial, l'enseignement de l'allemand fut abandonné. Mais ce ne fut pas la seule conséquence de la Première Guerre mondiale. Les Africains changèrent la vision idéaliste qu'ils pouvaient avoir des Européens. Ils exigèrent de meilleures formations, équivalentes à celles suivies par les Européens par exemple. Mais les Britanniques donnaient peu d'aide pour les écoles en général, et les écoles des missions en particulier puisqu'en 1924, la commission PhelpsStokes ${ }^{21}$ publia les résultats d'une grande enquête menée sur l'ensemble du protectorat d'Afrique de l'Est anglaise. En effet, pour l'année 1922-1923, le gouvernement britannique dépensa $f, 18.140$ pour l'éducation, soit environ $1 \%$ des dépenses totales pour la colonie. De plus, on constate une baisse dans ce maigre budget puisque un an plus tard, pour l'année 1923-1924, le gouvernement britannique dépensa $f, 13.156$ pour l'éducation, soit moins de $1 \%$ des dépenses totales pour la colonie du Tanganyika Territory, alors que le recensement de la population de 1921 compte 2.500 Européens, 15.000 Asiatiques et 4.000.000 d'Africains (800.000 ayant l'âge d'aller à l'école) (Jones,

\footnotetext{
20 « (...) doit aspirer par tous les moyens à ce que les indigènes apprennent l'allemand comme langue principale et langue de communication, car il s'agit du plus clair signe de pouvoir ».

${ }^{21}$ La commission Phelps-Stokes fut établie en 1911 dans l'Etat de New-York, reliant les chefs et organisations émergentes en ce qui concerne plus spécifiquement l'Amérique et l'Afrique. A ses débuts, elle s'intéressa beaucoup à l'éducation des Indiens d'Amérique ainsi que dans les années 20 à 60 à l'Afrique, et notamment à l'Afrique de l'Est. Elle joua un grand rôle au niveau de l'éducation sous le mandat britannique de l'Afrique orientale anglaise, privilégiant essentiellement l'agriculture et les travaux pratiques (Jones, 1921). En effet, elle croyait qu'une éducation pratique plutôt qu'intellectuelle permettrait de développer le potentiel agricole du territoire, dans la mesure où selon elle : « (...) the African is a peasant farmer at heart and should be trained rather than educated. » [i.e. "L'Africain est au fond un paysan-fermier et doit être entraîné plus qu'éduqué »] (Mbilinyi, 1980).
} 
1925). Dans ces conditions, les missionnaires eurent des difficultés à embaucher du personnel qualifié, ce qui les gêna considérablement dans leur mission d'éducation des masses. De plus, la crise économique de 1929 n'encouragea pas la Grande-Bretagne à investir davantage dans l'éducation de ses sujets. En 1934, l'administration coloniale attribua 24,2 $£$ par élève européen en Afrique de l'Est contre 14 Shillings pour un élève africain (Lawuo, 1980). Cette répartition des fonds correspond à la vision profondément raciste de l'administration britannique, catégorisant les individus selon leur « race » avec au sommet les «Blancs», puis les «Indiens» et les «Arabes» et tout en bas de la classification les «noirs». Cette disproportion dans l'investissement éducatif entre les élèves africains et européens ne changea pas jusqu'à l'indépendance. C'est pour cette raison que les Africains s'organisèrent en Native Councils' ${ }^{22}$ pour organiser leur propre politique scolaire. Les missionnaires étaient débordés par l'étendue des zones à parcourir et par une démographie croissante. Cette politique extrêmement raciste priva, par conséquent, pendant très longtemps, le protectorat anglais d'Afrique de l'Est d'école supérieure pour les Africains, l'équivalent des lycées actuels. Même si la GrandeBretagne développa des partenariats entre les écoles d'Afrique de l'Est - Kenya, Ouganda et Tanganyika ${ }^{23}$-, mais également entre l'Afrique de l'Est et la GrandeBretagne. Cette politique excluait les missions de leur rôle éducatif au sein de l'organisation coloniale.

Les missions étaient alors là pour jouer un rôle social sans vraiment être valorisées par la colonie britannique. Les Britanniques, qui pourtant étaient bien représentés au Tanganyika Territory avec trois sociétés missionnaires (London Missionary Society, Church Missionary Society et Universities' Mission to Central Africa), négligèrent la timide collaboration entreprise à la fin de la colonisation allemande entre les missionnaires et l'administration coloniale. Le système des fonds privés était beaucoup plus développé en Grande-Bretagne et aux Etats-Unis, ce qui permettait de financer des commissions, comme la commission Phelps-Stokes, mais aussi de faire d'importantes donations aux organismes missionnaires. Les congrégations religieuses ne devaient alors plus compter sur des subventions publiques mais privées pour subsister, des subventions qu'elles devaient chercher en Europe. Quant aux autres missions européennes, elles étaient encore moins reconnues et moins susceptibles de lever des fonds. Le soutien prévisible entre les deux grands acteurs de l'éducation formelle, en Afrique de l'Est, ne s'était donc pas développé.

Après plusieurs années où le pouvoir colonial britannique ne se manifesta pas en terme de politique scolaire et linguistique au Tanganyika Territory, il entreprit une politique plus globale en décidant d'instituer le kiswahili comme langue commune à l'ensemble de ses colonies ou protectorats d'Afrique de l'Est, à savoir l'Ouganda, le Kenya, Zanzibar et le Tanganyika. Le kiswahili était la langue d'enseignement à l'école

\footnotetext{
${ }^{22}$ Les Native councils signifient littéralement des conseils autochtones. Il s'agit ici de comités constitués de locaux africains qui discutent ensemble sur des sujets de société et tentent de trouver des solutions, sans faire appel à l'administration coloniale britannique.

${ }_{23}$ Il existait entre les différentes colonies d'Afrique de l'Est britanniques des accords pour envoyer les meilleurs éléments du Tanganyika dans les lycées professionnels et techniques d'Ouganda et du Kenya. La connaissance de l'anglais et du kiswahili permettait ce type d'échanges, même si cela ne concernait que très peu d'élèves compte tenu de la faiblesse démographique de la population tanganyikaise.
} 
primaire et dans les premiers échelons de l'administration. Malgré les nombreux travaux des missionnaires allemands, les Britanniques organisèrent en juin 1928 le Comité sur la langue territoriale qui se déroula à Mombasa, au Kenya, dans le but de normaliser un kiswahili standard. Le dialecte de Zanzibar, l'un des plus anciens, le kiunguja, fut choisi comme base à la standardisation du kiswahili dans la mesure où les langues swahilies étaient traditionnellement utilisées comme langue seconde sur le continent et comme langue première sur la côte et l'archipel de Zanzibar, espaces densément peuplés (Whiteley, 1969).

\section{La préparation à l'indépendance : la langue comme élément clé d'une identité nationale nouvelle}

Après la Seconde Guerre mondiale, l'Empire britannique devait se préparer à l'indépendance de ses colonies. Or, dans la mesure où le Tanganyika n'a jamais été une réelle colonie de peuplement, ni une colonie d'exploitation, se séparer de ce territoire était perçu comme moins douloureux. Par conséquent, les volontés d'indépendance formulées par l'association politique chef de file de la lutte anticoloniale, Tanganyika African Association (TAA) ${ }^{24}$, puis à partir de 1954 par la Tanganyika African National Union (TANU) furent entendues. Le slogan de la TANU, Uburu na umoja, qui signifie «Liberté et Unité » était clair : l'accès à l'indépendance était en ligne de mire - l'association était reconnue comme tel auprès des Nations-Unies. La langue de communication utilisée par le mouvement anticolonial était de fait le kiswahili, maitrisé par la quasi-totalité des habitants du Tanganyika à la fin des années 1950. Julius Nyerere, leader de la TANU et professeur dans un lycée missionnaire anglais, était conscient de l'importance de l'éducation et des langues; il avait aussi saisi combien la promotion du kiswahili par les colonisateurs avait contribué à constituer une identité commune, nationale au Tanganyika. Il devenait donc crucial que le kiswahili soit parlé et écrit par tous, peuple et élites.

Nyerere choisit donc le kiswahili pour être la langue officielle du Tanganyika indépendant, puis en 1964 de la Tanzanie, afin de dépasser les clivages ethniques, en plus de l'anglais. L'unification et la standardisation de la nouvelle langue nationale se poursuivirent avec l'Institute of Swabili Research de l'Université de Dar es-Salaam, et enfin le Baraza la Kiswahili la Taifa, organe officiel tanzanien. L'objectif à terme était de supplanter la langue coloniale, l'anglais, qui s'imposait déjà au parlement. Parce que le kiswahili ne favorisait aucune ethnie, il fut aisément accepté par la société. S'affranchir de la présence coloniale, c'était aussi la langue du colonisateur, sachant que «les langues constituent les organes des modes de penser et de ressentir propres aux nations » (von Humboldt, 1821). L'esprit, la pensée et la conscience sont liés de manière indéniable à la langue. Dans l'esprit des élites indépendantistes de l'époque, le Tanganyika ne pouvait pas, par conséquent, conserver l'emploi de la langue anglaise.

${ }^{24}$ Julius Nyerere était le président de la TAA en 1953, puis fonda la TANU en 1954. 
À la différence d'autres pays africains anciennement colonisés qui ont conservé comme langue véhiculaire la langue coloniale, le choix du kiswahili au Tanganyika puis en Tanzanie après l'indépendance est bien une particularité à souligner.

\section{Conclusion}

En conclusion, les politiques linguistiques en Afrique de l'Est allemande puis au Tanganyika britannique forment bien une exception vis-à-vis des autres colonies africaines, aussi bien françaises, qu'anglaises ou portugaises. L'introduction d'une langue africaine au niveau de tout un territoire et son passage de l'écriture arabe à l'écriture latine sont inédits. Le travail mené d'une part par les missionnaires mais aussi par les administrateurs coloniaux est conséquent; il a permis le succès de cette entreprise qui fut poursuivie par les Britanniques puis par le gouvernement indépendant tanganyikais, fier d'avoir une langue nationale africaine. C'est pourquoi à partir de 1965, les campagnes politiques, l'éducation, les séances de tribunaux en première instance, et de nombreux autres secteurs se font en kiswahili. On assiste alors à un second processus de swahilisation de la société tanzanienne. Puis à partir de 1970, le parlement national siège dans cette même langue. Dans les années 1980, l'enseignement secondaire ne se fait plus en anglais mais entièrement en kiswahili. En conséquence, les enfants parlent de moins en moins la langue de leurs parents, de leurs grands-parents, permettant au kiswahili de devenir la première langue, la langue nationale.

Le kiswahili est aussi la langue nationale du Kenya, de la République démocratique du Congo, de l'Ouganda et des Comores ainsi que la langue véhiculaire au Rwanda, au Burundi, au Mozambique, au Malawi, en Zambie et en Somalie. On compte actuellement entre 5 et 10 millions de personnes de langue maternelle swahilie et 40 à 60 millions de locuteurs pour qui le kiswahili est la seconde langue.

Aujourd'hui, le kiswahili s'est enrichi de diverses influences européennes allemande, portugaise, française et anglaise - mais aussi perse. Elle se modernise et évolue. De nombreux auteurs contemporains écrivent en kiswahili, notamment en Tanzanie et au Kenya. La présence du kiswahili sur la toile est également importante. Considérée comme la deuxième langue d'Afrique après l'arabe, le kiswahili est promis à un bel avenir! (Ricard, 2009).

Cependant, certains linguistes déplorent la disparition de dizaines de dialectes dans cette région où le kiswahili monopolise toute l'attention aussi bien dans la rue et la presse, qu'au niveau des travaux menés par les chercheurs. La richesse linguistique est alors en grand danger puisque la plupart de ces dialectes ne sont pas écrits. «Un vieillard qui meurt, c'est une bibliothèque qui brûle $»^{25}$.

\footnotetext{
${ }^{25}$ Proverbe malien reprit par l'écrivain d'origine malienne Amadou Hampaté Ba.
} 


\section{BIBLIOGRAPHIE}

\section{Sources:}

Masimulizi na mafundisho. Kitabu cha kwanæa, Mission Lwandai, 1931.

Acker, P. Amandus, "Die Aufgabe der katholischen Mission in den Kolonien», in Jabrbuch über die deutschen Kolonien, 2. Jg., Essen, 1909.

Archives des Pères du Saint-Esprit, 2K1.

Bundesarchiv, DKG, Berlin, Bd. Nr. 977.

Davesne, André, Mamadou et Bineta apprennent à parler français, cours de langage à l'usage des écoles africaines, classes de débutants et cours préparatoires, livre de maitre, Paris, ca. 1931.

Davesne, André, Mamadou et Bineta apprennent à lire et à écrire : syllabaire à l'usage des écoles africaines, classes de débutants et cours préparatoires, livre de maittre, Paris, ca. 1931.

Jones, Thomas Jesse, Educational adaptations report of ten years' work of the Phelps'-Stocks Fund 1910-1920, Phelps-Stokes Fund, New York, 1921.

Jones, Thomas Jesse, Education in East Africa, Phelps-Stokes Fund, New York, 1925.

König (von), Bernhard, «Die Eingeborenen-Schulen in den deutschen Kolonien Afrikas und der Südsee », in : Koloniale Rundschau, 1, 1913.

Mainhof, Carl, "Die Bedeutung des Studiums der Eingeborenensprachen für die Kolonialverwaltung », in Verbandlungen des Deutschen Kolonial-Kongresses 1905 zu Berlin, Berlin, 1906, pp. 343-363.

Mirbt, Carl, « Die Schulen für Eingeborenen in den deutschen Schutzgebiete », in Kolonial Monatsblätter, 16, 1914.

Schmidlin, Josef, Die katholische Missionen in der deutsche Schutzgebieten, Aschendorffsche Verlag, Münster, 1913.

Tanzanie National Archives, «MPAPUA an Gouvernement», 26.10.1901, in TNA, G $9 / 24$.

\section{Littérature secondaire:}

Calvet, Louis-Jean, Linguistique et colonialisme, petit traité de glottophagie, Paris, Payot, 1974.

Cuche, Denys, La notion de culture dans les sciences sociales, Paris, La Découverte, 2001.

Eggert, Johanna, Missionsschule und sozialer Wandel in Ostafrika. Der Beitrag der deutschen evangelischen Missionsgesellschaften zur Entwicklung des Schulwesens in Tanganyika 18911939, Bielefeld, 1970.

Gründer, Horst, Christliche Mission und deutscher Imperialismus. Eine politische Geschichte ibrer Beziebungen wäbrend der deutschen Kolonialzeit (1884-1914) unter besonderer Berücksichtigung Afrikas und Chinas. Paderborn, 1982.

Humboldt (von), Wilhelm, «Über den Einfluss des verschiedenen Charakters der Sprachen auf Literatur und Geistesbildung », in Gesammelte Schriften vol. 7, traduit de "De l'influence 
de la diversité de caractère des langues sur la littérature et la culture de l'esprit », in Sur le caractère national des langues et autres écrits sur le language (pp. 120-129), D. Thouard, Paris, Seuil, 2000.

Lawuo, Z.E., Education and social change in a rural community. A study of colonial education and local response among the Chagga between 1920 and 1945, Dar es-Salam, 1980.

Markmiller, Anton, "Die Erziehung der Negersarbeit”. Wie die koloniale Pädagogik afrikanische Gesellschaften in die Abhängigkeit führte, Berlin, Dietrich Reimer Verlag, 1995.

Mbilinyi, Marjorie, « African education during the British colonial period 1919-1961 », in Kaniki, Martin H.Y., (ed.), Tanzania under colonial rule, Londres, Longman, 1980.

Niesel, Hans-Joachim, Kolonialverwaltung und Missionen in Deutsch-Ostafrika 1890-1914, Fulda, 1971.

Paas, Steven, Jobannes Rebmann: A Servant of God in Africa before the Rise of Western Colonialism, Nuremberg, Verlag für Theologie and Religionswissenschaft, 2011.

Ploeg (van der), Arie J., "Education in Colonial Africa: The German Experience », in Comparative Education Review, 21, Pittsburg, 1977.

Porte, Rémy, La conquête des colonies allemandes. Naissance et mort d'un rêve impérial, Paris, Belin, 2006.

Ricard, Alain, Le Kiswabili, une langue moderne, Paris, Karthala, 2009.

Sapir, Edward, Culture et personnalité, trad. Christian Baudelot et Pierre Clinquart, Paris, 1995.

Schäppi, Franz Solan, Die katholische Missionsschule im ebemaligen Deutsch-Ostafrika, Paderborn, 1937.

Spellmeyer, Hans, Deutsche Kolonialpolitik im Reichstag, Stuttgart, 1931.

Tetzlaff, Rainer, «Die Mission im Spannungsfeld zwischen kolonialer Herrschaftssicherung und Zivilisationsanspruch in Deutsch-Ostafrika ", in Bade, Klaus (Hrsg.), Imperialismus und Kolonialmission. Kaiserliches Deutscbland und koloniales Imperium, Stuttgart, 1984.

Whiteley, Wilfred, Swabili: The rise of a national language, London: Methuen (Series: Studies in African History), 1969. 